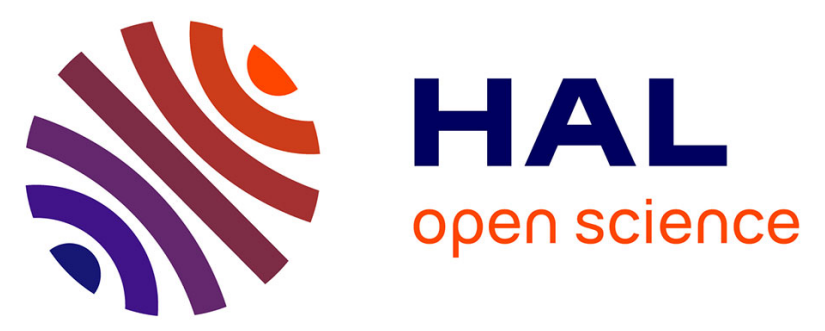

\title{
Étude cinématique des événements binaires et ternaires obtenus par bombardement de cibles d'U, Th, Bi, Pb, Au par des deutons de 2,1 GeV
}

\author{
M. Debeauvais, G. Gheysari, F. Rahimi, J. Ralarosy, G. Remy, J. Tripier
}

\section{- To cite this version:}

M. Debeauvais, G. Gheysari, F. Rahimi, J. Ralarosy, G. Remy, et al.. Étude cinématique des événements binaires et ternaires obtenus par bombardement de cibles d'U, Th, $\mathrm{Bi}, \mathrm{Pb}, \mathrm{Au}$ par des deutons de 2,1 GeV. Journal de Physique, 1978, 39 (7), pp.740-749. 10.1051/jphys:01978003907074000 . jpa00208809

\section{HAL Id: jpa-00208809 https://hal.science/jpa-00208809}

Submitted on 1 Jan 1978

HAL is a multi-disciplinary open access archive for the deposit and dissemination of scientific research documents, whether they are published or not. The documents may come from teaching and research institutions in France or abroad, or from public or private research centers.
L'archive ouverte pluridisciplinaire HAL, est destinée au dépôt et à la diffusion de documents scientifiques de niveau recherche, publiés ou non, émanant des établissements d'enseignement et de recherche français ou étrangers, des laboratoires publics ou privés. 


\title{
ÉTUDE CINÉMATIQUE DES ÉVÉNEMENTS BINAIRES ET TERNAIRES OBTENUS PAR BOMBARDEMENT DE CIBLES D'U, Th, Bi, Pb, Au PAR DES DEUTONS DE 2,1 GeV
}

\author{
M. DEBEAUVAIS, G. GHEYSARI $(*)$, F. RAHIMI, J. RALAROSY, G. REMY et J. TRIPIER
}

Centre de Recherches Nucléaires, S.A.D.V.I., 67037 Strasbourg Cedex, France

(Reçu le 8 juillet 1977, révisé le 24 février 1978, accepté le 24 mars 1978)

\begin{abstract}
Résumé. - Une étude cinématique de la fission binaire et ternaire induite par des deutons de $2,1 \mathrm{GeV}$ sur des cibles $\mathrm{U}, \mathrm{Th}, \mathrm{Pb}, \mathrm{Bi}$ et $\mathrm{Au}$ est réalisée à partir des caractéristiques géométriques des traces des fragments des événements enregistrés dans un détecteur solide de traces en géométrie $4 \pi$. Moyennant certaines hypothèses, on établit la distribution en rapport de masse et en énergie des fragments. On a pu mettre en évidence qu'une partie des événements binaires dont l'angle projeté sur le plan perpendiculaire à la trajectoire de la particule incidente est différent de $180^{\circ}$, procèdent d'un même type de désintégration que les événements ternaires. De même, l'analyse cinématique des événements ternaires montre une certaine analogie avec le phénomène connu de la tripartition de noyaux lourds comme le californium par exemple, et pourrait expliquer la production d'une partie des isotopes légers et lourds déficients en neutrons attribués à de la fragmentation par les radiochimistes.
\end{abstract}

\begin{abstract}
A kinetic study of binary and ternary fission induced by $2.1 \mathrm{GeV}$ deuterons incident on $\mathrm{U}, \mathrm{Th}, \mathrm{Pb}, \mathrm{Bi}$ and $\mathrm{Au}$ targets has been made by obtaining the geometrical characteristics of events registered in a $4 \pi$ solid state track detector. Using some hypothesis the distribution of the mass ratios and the total kinetic energy of the fragments were measured. We thus established that part of the binary events whose projected angles on the plane perpendicular to the incident deuteron differ by $180^{\circ}$ arise from the same type of disintegrations as the ternary events. The analytical determination of the ternary events also shows an analogy with the tripartition of heavy nuclei such as for example californium and can also explain the production of part of the heavy and light neutron deficient isotopes, which the radiochemists attribute to fragmentation.
\end{abstract}

1. Introduction. - L'étude est réalisée à l'aide d'un détecteur visuel solide le makrofol. Dans un article précédent [1], nous avons donné les conditions expérimentales de cette expérience ainsi que les résultats concernant les sections efficaces de fission double et triple de $\mathrm{l}^{\prime} \mathrm{U}, \mathrm{Th}, \mathrm{Bi}, \mathrm{Pb}$ et $\mathrm{Au}$ induites par des deutons de 2,1 GeV. Dans ce travail nous ferons appel aux caractéristiques géométriques des traces de fission enregistrées dans ce détecteur $4 \pi$, longueur, angle, qui nous permettent de déduire, moyennant certaines hypothèses supplémentaires, les masses et énergies des fragments pour les différentes cibles bombardées. Ces résultats permettront de faire un nouveau pas en avant vers la connaissance $\mathrm{du}$ phénomène de fission produit par des particules de haute énergie.

(*) Faculté, Université des Sciences, Ahwaz, Iran.
2. Techniques expérimentales. - Les caractéristiques et le mode de développement des détecteurs visuels de traces ont déjà été décrits [2], signalons seulement que les cibles sont évaporées directement sur le polycarbonate ou makrofol et leurs épaisseurs se situent aux alentours de 50 à $100 \mu \mathrm{g} / \mathrm{cm}^{2}$ ce qui n'affecte ni la longueur d'enregistrement des traces de fission, ni l'énergie de ces dernières. Les trois différents types d'événements détectés et étudiés sont représentés sur la figure 1 . Seuls les deux derniers types d'événements font l'objet de cette étude. Les traces laissées par les fragments sont mesurées à l'aide d'un microscope optique avec un grossissement d'environ 3000 . On détermine ainsi pour chaque trace la longueur projetée sur le plan détecteur, son enfoncement et l'angle entre les projections des deux ou trois traces corrélées. Les incertitudes de mesures sont : $\sim 0,5 \mu$ sur la mesure de la projection, environ $1 \mu \mathrm{m}$ sur l'enfoncement, de 0 à $2^{\circ}$ sur l'angle azimutal. 

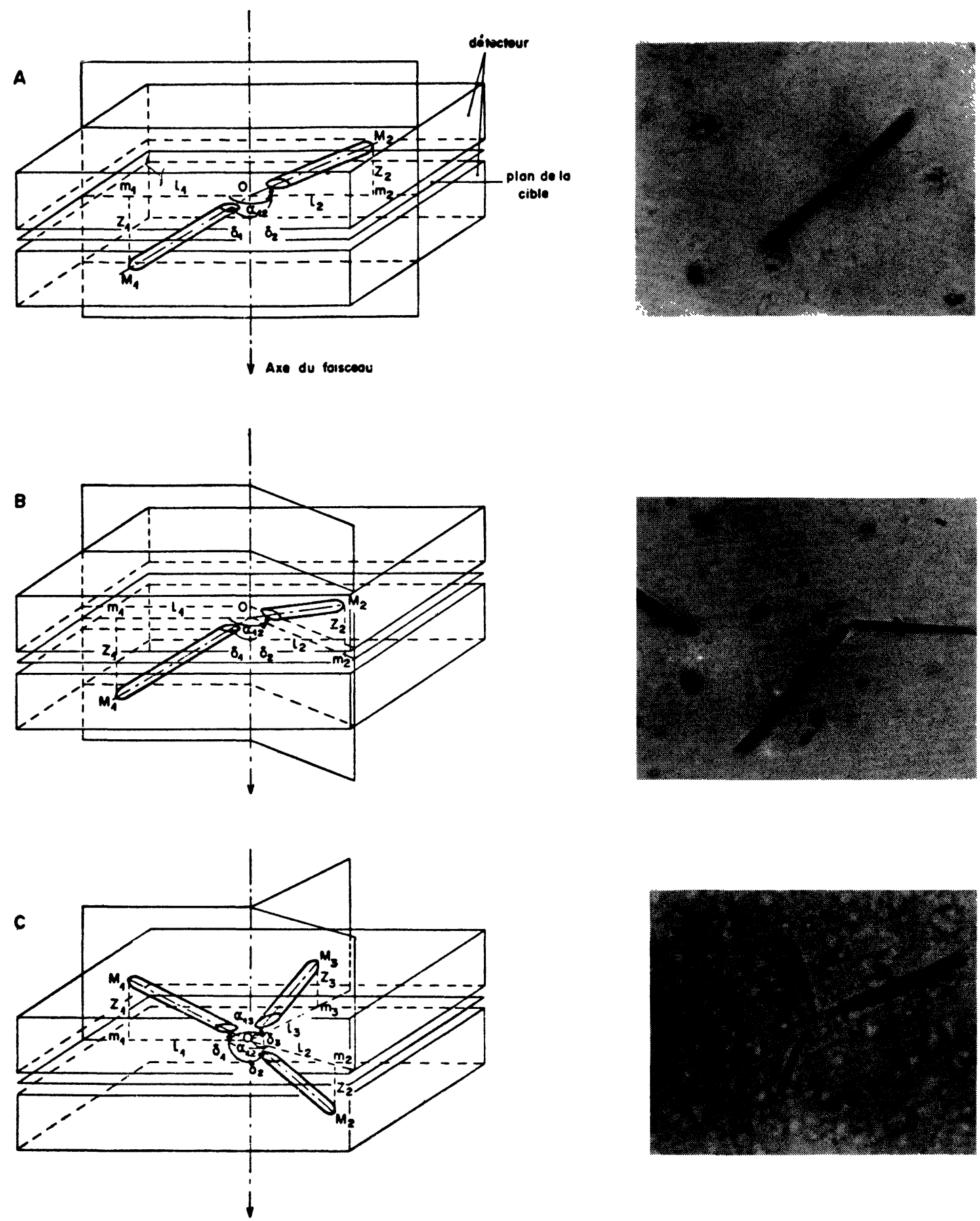

Fig. 1. - Confirmation dans l'espace des événements caractéristiques issus de la fission de l'U bombardé par des deutons de $2.1 \mathrm{GeV}$ enregistrés dans le makrofol.

[Typical fission events observed in Makrofol after bombarding $\mathrm{U}$ target with $2.1 \mathrm{GeV}$ deuterons.]

3. Propriétés géométriques des traces dues aux fragments. - 3.1 PARCours. - La mesure de la longueur projetée ainsi que de l'enfoncement de la trace par rapport au plan du détecteur nous permet de déterminer la longueur exacte de chaque trace. Cette longueur correspond au parcours intégral dans le détecteur du fragment correspondant. Pour les événements binaires nous avons sélectionné : a) $R_{1}$ et $R_{2}$ longueurs des traces orientées vers l'avant et l'arrière respectivement;

b) $R_{\mathrm{L}}$ traces les plus longues et $R_{\mathrm{H}}$ traces les plus courtes.

Dans chaque cas, nous avons établi les distributions des longueurs de traces et les valeurs moyennes $\left(\bar{R}_{1}, \bar{R}_{2}, \bar{R}_{\mathrm{L}}, \bar{R}_{\mathrm{H}}, \bar{R}\right)$ ainsi que les déviations $\sigma$ standard 
TABLEAU I

Résultats pour les fissions binaires

[Results concerning binary fission]

\begin{tabular}{lcccccccccccc}
\multicolumn{1}{c}{ Cible } & $\bar{R}$ & $\bar{R}_{1}(\mu)$ & $\sigma R_{1}$ & $\bar{R}_{2}(\mu)$ & $\sigma R_{2}$ & $\bar{R}_{\mathrm{H}}(\mu)$ & $\sigma R_{\mathrm{H}}$ & $\bar{R}_{\mathrm{L}}(\mu)$ & $\sigma R_{\mathrm{L}}$ & $\sigma \alpha_{12}$ & $\bar{\delta}$ (degré) & $\sigma_{\delta}$ \\
\multicolumn{1}{c}{} & - & - & $\overline{19}$ & - & - & - & - & - & - & - & - & - \\
$\mathrm{Au}^{197}$ & 13,2 & 13,8 & 3,6 & 12,6 & 3,7 & 10,5 & 2,5 & 15,6 & 3,4 & 19,5 & 168,9 & 16,8 \\
$\mathrm{~Pb}^{207}$ & 13,9 & 14,6 & 4,0 & 13,1 & 3,8 & 11,2 & 2,7 & 16,5 & 3,3 & 15,8 & 170,0 & 14,0 \\
$\mathrm{Bi}^{208}$ & 15,2 & 16,5 & 4,6 & 14,0 & 4,0 & 12,3 & 2,6 & 18,2 & 4,1 & 16,0 & 169,0 & 16,0 \\
$\mathrm{Th}^{232}$ & 15,8 & 16,4 & 2,8 & 15,2 & 2,9 & 13,7 & 2,2 & 17,8 & 2,3 & 11,4 & 173,2 & 11,6 \\
$\mathrm{U}^{238}$ & 16,0 & 16,6 & 2,9 & 15,3 & 3,1 & 14,1 & 2,4 & 17,9 & 2,6 & 10,0 & 175,0 & 9,0 \\
$\mathrm{U}^{235}$ n.Th & 17,9 & 17,8 & 2,5 & 18,0 & 2,4 & 16,0 & 1,6 & 20,0 & 1,5 & 3,0 & 180,0 & 2,0
\end{tabular}

$\left(\sigma_{R_{1}}, \sigma_{R_{2}}, \sigma_{R_{\mathrm{L}}}, \sigma_{R_{\mathrm{H}}}\right)$ correspondantes. Les quantités sont reportées sur le tableau I. Sur le même tableau, nous montrons également les valeurs des parcours relatives à la fission de l'U induite pas des neutrons thermiques et déterminées à l'aide de la même technique. Tous les résultats présentés se rapportent à des événements binaires dont les 2 traces ont des longueurs supérieures à $5 \mu$. Le parcours intégral $R_{\mathrm{i}}$ est déterminé à partir de la méthode des développements séquencés. Seuls seront retenus les événements dont la longueur de trace $R_{\mathrm{i}}$ ne varie pas entre le développement standard $(40 \mathrm{~min}$.) et la seconde phase de développement $(40+50 \mathrm{~min}$.).

Notons que seuls 2 à $3 \%$ d'événements binaires verront leur longueur de trace augmentée d'un développement à l'autre d'un facteur allant jusqu'à $30 \%$.

3.2 Ängles. - On mesure l'angle $\alpha_{i j}$, entre les projections des traces corrélées sur le plan $x y\left(\alpha_{i j} i \neq j\right.$, $i, j, \ldots=1,2, \ldots, n ; n=$ nombre de fragments).

Nous calculons l'angle $\delta_{i}$ entre l'axe $Z^{\prime} Z$ et la direction de la trace. On détermine donc les distributions des angles $\alpha_{12}$ et $\delta=\delta_{1}+\delta_{2}$; leurs valeurs moyennes ainsi que les déviations standard correspondantes. Les valeurs figurent sur le tableau I. On indique à titre de référence les valeurs relatives à la fission thermique.

3.3 Discussion. - Les valeurs moyennes de l'angle $\delta$ montrent que l'entraînement du noyau fissionnant est préférentiellement dirigé vers l'avant et que le transfert d'impulsion est le plus élevé pour les cibles les plus légères. L'effet de ce transfert d'énergie cinétique au noyau de recul se traduit par des longueurs de traces supérieures dans le cas des fragments émis dans l'hémisphère avant $R_{1}$ que dans l'hémisphère arrière $R_{2}$, quelle que soit la cible.

Le parcours moyen $\bar{R}$ des fragments est une fonction croissante de la masse de la cible. On remarque également que la valeur moyenne des parcours des fragments de fission de l'U induite à $2,1 \mathrm{GeV}$ par des deutons ou par des protons de même énergie est pratiquement identique mais est inférieure à la valeur correspondante de la fission de l'U induite par des neutrons thermiques. Ces résultats semblent compatibles avec l'hypothèse de la répulsion purement coulombienne des fragments issus d'un noyau de recul moyen, sensiblement plus léger que le noyau cible - du fait de l'évaporation de particules dans le premier stade de la réaction - l'énergie d'excitation du système ne contribuant que peu à l'énergie cinétique de translation. Notons également que le rapport $\mathrm{du}$ parcours du fragment léger au fragment lourd augmente lorsque la masse de la cible diminue, ce qui caractérise une fission d'autant plus symétrique que la masse de la cible diminue.

Nous avons porté une attention particulière à l'étude de la distribution de l'angle projeté $\alpha_{12}$. En effet, la fission binaire induite par des particules de basse énergie et passant par la formation d'un noyau composé ne peut être considérée comme une réaction à 2 corps que si les effets des émissions de neutrons prompts ou retardés n'ont pratiquement aucune influence sur les propriétés cinématiques des deux fragments de fission émis. Dans cette approximation, il existe un plan de réaction défini par la direction du faisceau incident et l'axe de la fission dans le système du centre de masse dans lequel les projections des 2 traces sur le plan perpendiculaire à la direction du faisceau, forment un angle strictement égal à $180^{\circ}$. C'est le cas de la fission de l'U par des neutrons thermiques. La distribution de l'angle $\alpha_{12}$ est d'allure semi-gaussienne $180^{\circ}$ (écart standard $2^{\circ} 5$ ) (Fig. 2a). Sur cette figure, nous avons reporté les distributions normalisées $\mathrm{d} N / \mathrm{d} \alpha_{12}$ de l'angle $\alpha_{12}$ et calculé les déviations standard de distribution qui figurent sur le tableau I. Sur la figure $2 b$, nous avons reporté cette déviation standard en fonction du paramètre $Z^{2} / A$ de la cible. La pente moyenne de la droite ajustée est de 0,22\%/UMA. Les travaux de Brandt et coll. [3] ainsi que ceux de Remsberg et coll. [4] indiquent également un élargissement de la corrélation angulaire (projetée notamment) de la fission binaire induite par des protons lorsqu'on passe d'une cible lourde comme l'U à une cible plus légère telle que l'Au.

L'apparition, à haute énergie, d'événements caracté- 

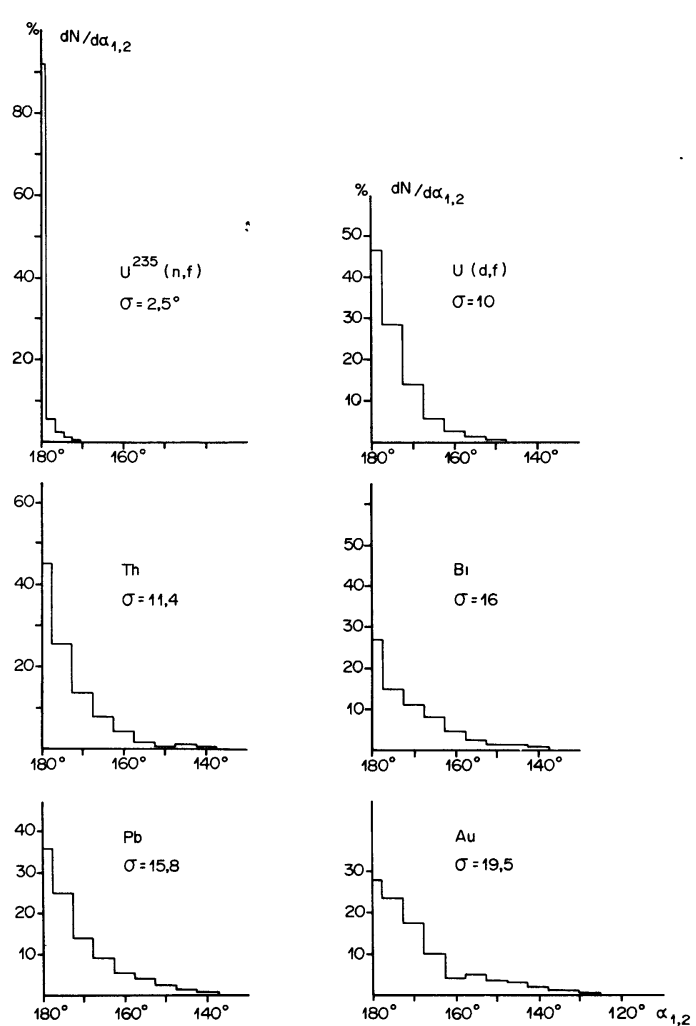

FIG. 2a. - Distribution de l'angle projeté ${ }_{q 12}$ entre les 2 traces sur le plan perpendiculaire à la direction du deuton incident.

[Distribution of the projected angle $\alpha_{12}$ between the 2 fragments on the plan perpendicular to the incident deuteron beam.]

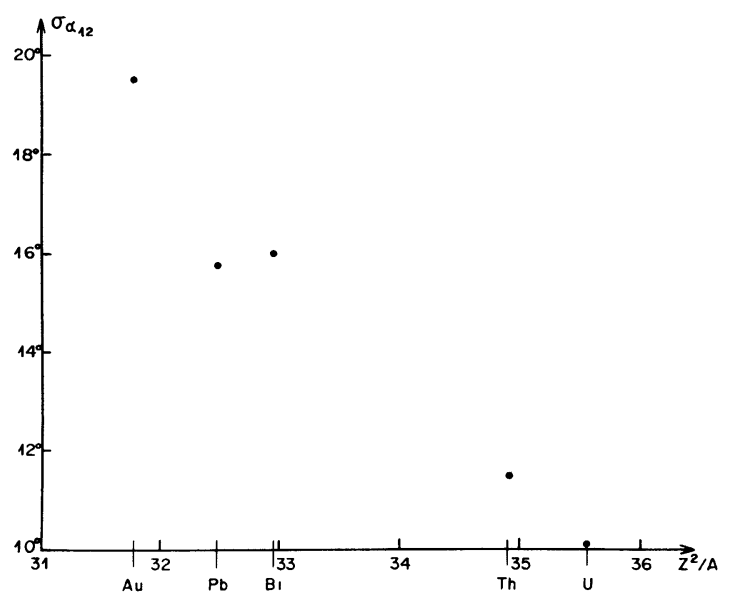

FIG. $2 b$. - Déviation standard de l'angle $\cdots 12$ en fonction du paramètre $Z^{2} / A$ de la cible.

[Standard deviation of the angle $\alpha_{12}$ as a function of the target parameter $Z^{2} / A$.]

risés par des angles projetés $\alpha_{12}$ sensiblement inférieurs à $180^{\circ}$ indique que les événements considérés mettent en jeu dans le système du laboratoire des impulsions transverses non négligeables par rapport à l'axe du faisceau. Ces événements sont généralement considérés par les auteurs comme étant de la fission binaire classique en vol.
Cependant le travail de Rémy [5] étudiant la fission de l'U et du $\mathrm{Pb}$ induite par des protons d'énergie variant entre 0,6 et $23 \mathrm{GeV}$ a montré, grâce à des considérations théoriques basées sur des résultats expérimentaux, que dans le cas des événements binaires, nous serions en présence de 2 types de réactions :

a) la fission en vol du noyau de recul en deux fragments seulement qui correspondrait à des événements dont l'angle $\alpha_{12}$ est voisin de $180^{\circ}$;

b) la désintégration du noyau de recul en 3 - éventuellement plus de 3 - fragments dont seuls les 2 fragments lourds seraient détectés sous forme de coïncidence et dont l'angle entre les 2 traces serait très différent de $180^{\circ}$.

Signalons que l'élargissement de la déviation standard $\alpha_{12}$ se fait dans le même sens que l'augmentation $\mathrm{du}$ rapport du nombre de fission triple au nombre de fission double [1] en fonction du paramètre $Z^{2} / A$ de la cible.

4. Etude cinématique. - L'étude cinématique complète de la fission à quelques $\mathrm{GeV}$ n'est possible. compte tenu des données expérimentales disponibles, qu'en faisant des hypothèses sur la masse et la charge du noyau fissionnant.

Dans une première étape, nous supposons que le - noyau fissionnant est identique au noyau composé. En fait, les calculs théoriques [6] et les résultats expérimentaux [4] font état de cascades pouvant atteindre plusieurs dizaines de nucléons dans le domaine d'énergie considéré. Les résultats que nous obtiendrons nous donneront donc des valeurs de masses et d'énergies toujours surestimées. Nous nous bornerons à présenter les résultats ne dépendánt que peu des hypothèses.

Pour obtenir les solutions en masse et en énergie des fragments de fission, nous faisons les hypothèses suivantes :

4.1 HypothÈSES. - 4.1.1 Identité du noyau de recul. - Nous admettons que le noyau de recul qui se divise en 2 ou 3 fragments est identique au noyau composé.

Nous appliquons les lois de conservation de la charge et de la masse pour ce noyau

$$
\begin{aligned}
& \sum_{i=1}^{n} Z_{i}=Z_{\mathrm{c}}+Z_{\mathrm{p}}=Z_{\mathrm{NC}} \\
& \sum_{i=1}^{n} M_{i}=M_{\mathrm{c}}+M_{\mathrm{p}}=M_{\mathrm{NC}}
\end{aligned}
$$

où $Z_{\mathrm{c}}, M_{\mathrm{c}}$ sont la charge et la masse de la cible et $\dot{Z}_{\mathrm{p}}, M_{\mathrm{p}}$ charge et masse du projectile, NC l'indice relatif au noyau composé, $n$ le nombre de fragments.

4.1.2 Dans l'hypothèse où les fragments enregistrés représentent intégralement la masse du noyau composé. l'impulsion transverse (composante perpendiculaire à 
la direction de la particule incidente) du système est strictement nulle.

A haute énergie, nous avons mis en évidence des événements binaires dont l'angle projeté $\alpha_{12}$ sur le plan perpendiculaire à la direction du deuton incident est sensiblement différent de $180^{\circ}$ et pour lesquels l'impulsion transverse ne peut être nulle. De même, certains événements ternaires font également apparaître une impulsion transverse importante. La méthode de résolution adoptée fait appel à la minimisation de la composante transverse de l'impulsion du noyau de recul. Cette hypothèse nous semble justifiée du fait que dans notre calcul nous n'avons retenu que les événements de fission dont l'angle $\alpha_{12}>175^{\circ}$.

4.1.3 L'hypothèse d'égale distribution de charge $(E C D)$. - Dans cette hypothèse, les fragments primaires de la fission ont même rapport de masse sur charge que le noyau fissionnant. Cette hypothèse est couramment admise dans l'étude de la fission à haute énergie.

\subsection{DONNÉES DONT ON DISPOSE. - 4.2.1 Géomé-} trie de l'événement.

a) Parcours $R_{i}$ des fragments ;

b) angle de chaque fragment avec la direction du deuton incident $\delta_{i}$ et la projection de l'angle entre les deux traces de fragment $\alpha_{i j}$ sur le plan perpendiculaire au faisceau.

4.2.2 Relation parcours énergie dans le makrofol pour tout ion lourd en fonction de sa masse et de sa charge [8].

4.2.3 Dérivée de ces relations, la masse minimale d'un ion associée à une longueur de trace pour un rapport $M / Z$ donné et à un temps de développement donné [2]. Cette condition permet de restreindre le nombre de combinaisons dans un domaine physique valable en fonction du détecteur et du développement utilisé ; elle est surtout utile dans le calcul des événements ternaires.

4.2.4 Les charges $Z_{\mathrm{c}}$ et $Z_{\mathrm{p}}$ et les masses $M_{\mathrm{c}}$ et $M_{\mathrm{p}}$ du noyau cible (c) et du projectile (p) respectivement.

4.3 MÉTHOde DE RÉSOlUTION D'UN ÉVÉNEMENT. Pour obtenir les distributions en énergie, masses et charges des fragments, nous effectuons les calculs au moyen d'un programme FORTRAN utilisant les paramètres et les hypothèses énoncés précédemment. Afin de n'obtenir qu'une seule solution, le programme de calcul examine toutes les combinaisons de masses possibles et ne retient que celle conduisant à l'impulsion transversale minimale. On peut calculer ensuite :

a) les énergies des fragments dans le système du laboratoire et dans le système du centre de masse du noyau composé hypothétique ;

b) le rapport de masse du fragment lourd au fragment léger pour les événements binaires ;

c) les angles entre les fragments dans le système du centre de masse dans le cas des événements ternaires.
4.4 RéSultats ET DisCUSSION. - Les résultats présentés ici se rapportent exclusivement aux événements binaires satisfaisant à la double inégalité suivante :

$$
0,1 \leqslant\left|\cos \delta_{i}\right| \leqslant 0,6
$$

et dont les longueurs de traces $R_{i}>5 \mu$ d'une part, et dont l'angle $\alpha_{12}>175^{\circ}$ d'autre part. Ce critère de sélection découle de la précision sur les mesures de longueur qui est la meilleure dans cet intervalle.

Nous présentons ici uniquement les résultats qui ne dépendent que très peu des hypothèses indiquées précédemment.

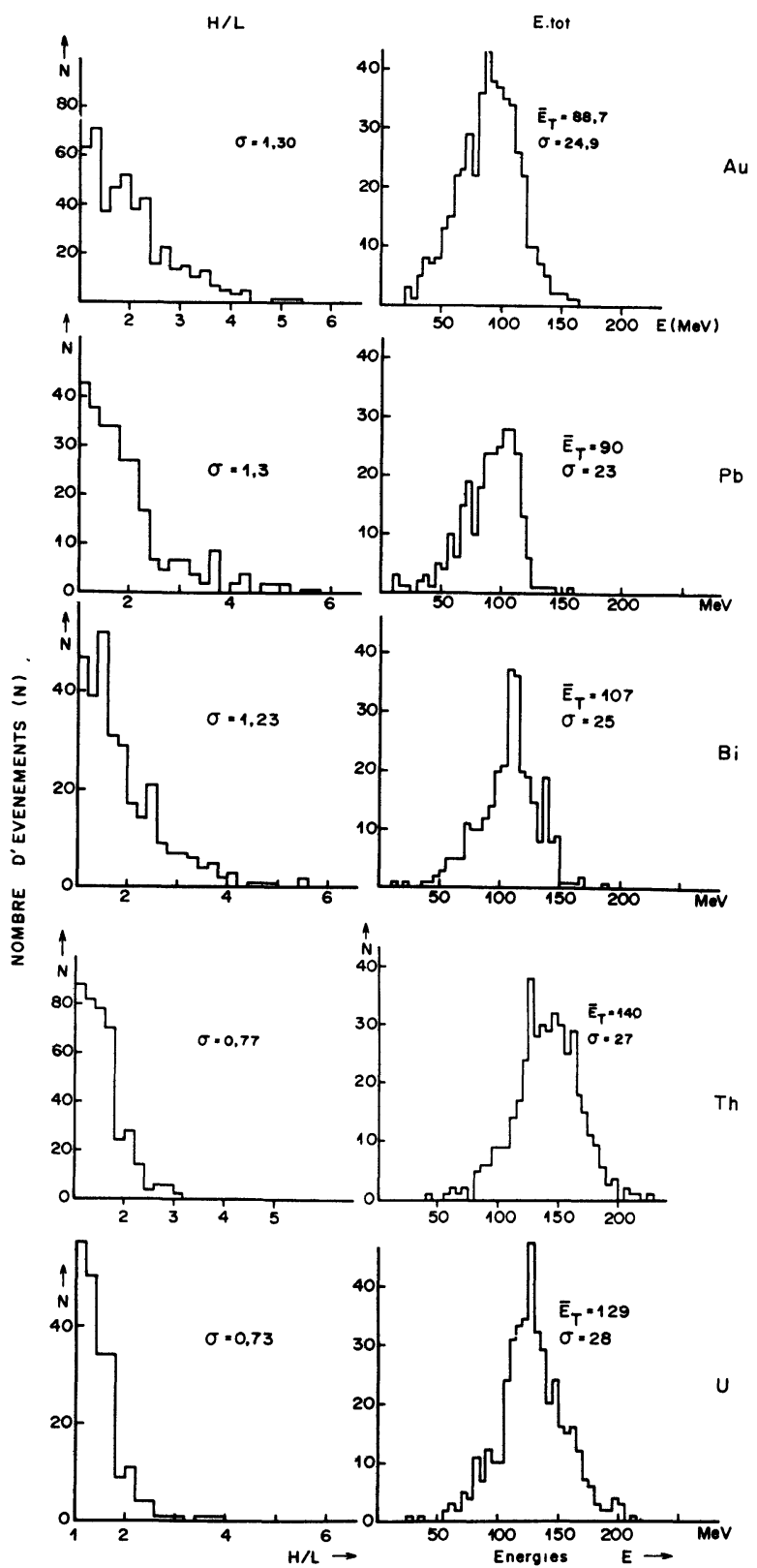

FIG. 3. - Distribution des énergies cinétiques totales dans le système du centre de masse des fragments de fission binaires de l'U, $\mathrm{Th}, \mathrm{Bi}, \mathrm{Pb}$ et $\mathrm{Au}$. Distribution des rapports de masse du fragment léger pour $\mathrm{U}, \mathrm{Th}, \mathrm{Bi}, \mathrm{Pb}, \mathrm{Au}$.

[Total kinetic energy distribution in the centre of mass system of $U$, $\mathrm{Th}, \mathrm{Bi}, \mathrm{Pb}$ and $\mathrm{Au}$ fission fragments. Mass yield of heavy to light fragment for $\mathrm{U}, \mathrm{Th}, \mathrm{Bi}, \mathrm{Pb}$ and $\mathrm{Au}$.] 
4.4.1 Fission binaire. - a) Energie cinétique totale. - La figure 3 représente les distributions d'énergie cinétique totale des fragments de la fission de l'U, Th, Bi, $\mathrm{Pb}$ et de l'Au dans le système du centre de masse (hypothétique) $E_{\mathrm{CMT}}$.

Bien que les valeurs découlent du calcul fait avec l'hypothèse du noyau composé, il faut signaler que l'énergie des fragments est déduite de leurs parcours et qu'elle n'est que peu dépendante des hypothèses. Une erreur de $1 \mu$ sur le parcours est traduite par une incertitude de $\sim 8 \mathrm{MeV}$ sur l'énergie cinétique du fragment. Les distributions de l'énergie cinétique totale des fragments pour les cibles étudiées ont une allure sensiblement gaussienne avec une déviation standard appréciable qui va en diminuant de l'uranium à l'or.

Les valeurs moyennes de l'énergie cinétique totale sont une fonction croissante de la masse de la cible, mais elles sont inférieures aux valeurs correspondant à la fission de ces noyaux à énergie intermédiaire [9].

\section{TABLEAU II}

Comparaison des énergies cinétiques totales de fission de $\mathrm{U}, \mathrm{Bi}$ et $\mathrm{Au}$ trouvées à différentes énergies de protons incidents.

[Total kinetic energy comparison of $\mathrm{U}, \mathrm{Th}, \mathrm{Bi}$, $\mathrm{Pb}$ and $\mathrm{Au}$ fission induced by protons of different incident energies.]

\begin{tabular}{cccc} 
& \multicolumn{4}{c}{ Cible } \\
Projectiles & \multicolumn{3}{c}{ Energie cinétique en MeV } \\
& $\mathrm{U}$ & $\mathrm{Bi}$ & $\mathrm{Au}$ \\
- & 165 & - & - \\
$\begin{array}{l}\text { Neutrons thermiques } \\
\text { Protons de } 156 \mathrm{MeV} \\
\quad \text { Kovalski [9] }\end{array}$ & $166 \pm 3$ & $154 \pm 3$ & $144 \pm 3$ \\
$\begin{array}{c}\text { Protons de 156 MeV } \\
\text { Peter [11] }\end{array}$ & & $145 \pm 10$ \\
$\begin{array}{c}\text { Protons de 2,9 GeV } \\
\text { Remsberg [7, 10] }\end{array}$ & $146 \pm 18$ & $121 \pm 22$ & \\
$\begin{array}{c}\text { Deutons de 2,1 GeV } \\
\text { Nos résultats }\end{array}$ & $129 \pm 28$ & $107 \pm 25$ & $90 \pm 23$
\end{tabular}

Dans le tableau II, nous comparons nos résultats avec les valeurs obtenues par différents auteurs [10,11] dans le système du laboratoire pour la fission de l'U, $\mathrm{du} \mathrm{Bi}$ et de l'Au induite par des protons de différentes énergies. Les valeurs de l'énergie cinétique totale à haute énergie sont plus basses qu'à énergie intermédiaire et peuvent s'expliquer de la manière suivante :

Les résultats expérimentaux à énergie intermédiaire [12] et haute énergie [7] ainsi que les résultats du calcul basé sur la théorie de la goutte liquide [13] montrent d'une part que l'énergie cinétique totale des fragments dans le système du centre de masse du noyau fissionnant est pratiquement indépendante de l'énergie d'excitation du noyau fissionnant, et d'autre part que cette énergie cinétique est essentiellement due à la répulsion coulombienne des deux fragments. Par conséquent, la charge du noyau fissionnant est un paramètre effectif. Evidemment, à haute énergie, le noyau fissionnant qui subit les processus de cascade et d'évaporation, possède une charge plus petite que celle obtenue à énergie intermédiaire. En effet, si l'on suppose pour l'U une évaporation de 20 neutrons et de 7 protons en accord avec les calculs de Hahn et Bertini [4] et les résultats de Remsberg et al. [7, 10], nous obtenons pour l'U une énergie cinétique totale de $154 \mathrm{MeV} \pm 33$ au lieu de $129 \mathrm{MeV} \pm 28$ pour la fission à partir du noyau composé. De même, si nous prenons pour l'Au comme noyau fissionnant $Z=76$ et $M=175$, soit une évaporation de 4 protons et 17 neutrons, l'énergie cinétique que nous obtenons est de $97 \mathrm{MeV} \pm 21$ au lieu de $90 \pm 23$ pour le noyau composé $Z=80, M=199$.

L'augmentation de la déviation standard de la distribution de l'énergie cinétique totale $\left(\sigma E_{\mathrm{CMT}}\right)$ en fonction de la masse de la cible peut être expliquée par les propriétés des noyaux lourds. Les calculs faits d'après la théorie de la goutte liquide indiquent que la déviation standard $\left(\sigma E_{\mathrm{CMT}}\right)$ est reliée aux fluctuations de la forme du noyau au point de scission autour d'une forme moyenne, et que la durée de transition du point selle au point de scission est d'autant plus petite que le noyau est plus léger.

La barrière de fission des noyaux très lourds $\mathrm{U}, \mathrm{Th}$ est basse, et à haute énergie, les noyaux issus d'interaction directe se distribuent sur un domaine très large de $Z$ et $M$, et de la température nucléaire ; presque tous les noyaux aux différentes températures nucléaires peuvent fissionner et le spectre d'énergie cinétique totale est large, tandis que pour les noyaux plus légers $\mathrm{Bi}, \mathrm{Pb}$ et $\mathrm{Au}$, la barrière de fission est plus haute, les noyaux fissionnants sont limités dans un domaine relativement petit et le spectre d'énergie est plus étroit.

b) Rapport de masse. - Les distributions des rapports de masses du fragment lourd au fragment léger sont représentées sur la figure 3. Les courbes ont une allure semi-gaussienne mais la déviation standard est plus élevée pour l'Au que pour l'U, ce qui indique une composante asymétrique plus importante pour les noyaux légers que pour les noyaux lourds. Pour mieux analyser l'aspect de la fission à haute énergie $2,1 \mathrm{GeV}$, nous avons classé les événements binaires en deux groupes bien distincts, les événements dont l'angle $\alpha_{12}$ est voisin de $180^{\circ}$, c'est-à-dire $175^{\circ}<\alpha_{12}<180^{\circ}$ (événement $\pi$ ) et ceux dont $\alpha_{12}$ est différent de $180^{\circ}$, c'est-à-dire $<175^{\circ}$ (événement $\not$ ) ).

Pourquoi faire cette distinction ?

Des expériences multiparamétriques classiques récentes faites par Remsberg [4] avec des protons de $2,9 \mathrm{GeV}$ sur des cibles d'U et de $\mathrm{Bi}$, il résulte que le mécanisme de fission induite à haute énergie est essentiellement le même qu'aux basses énergies.

Postkanzer, Butler et Hyde [16] utilisant un téléscope, et plus récemment Herz et coll. [14] avec du lexan comme détecteur, ont déterminé les spectres en 
énergie des isotopes de $Z$ inférieure à 40 éjectés d'une cible d'U bombardée par des protons de $5,5 \mathrm{GeV}$. Les résultats confirment les rendements élevés en fragments très légers trouvés antérieurement et montrent en particulier que dans l'hypothèse d'un mécanisme d'évaporation les hauteurs effectives des barrières coulombiennes devraient être réduites de $50 \%$ pour pouvoir ajuster les différents spectres en énergie. Parmi les interprétations possibles, ces auteurs suggèrent que les fragments considérés proviennent, soit d'une fission très asymétrique, soit du col d'un noyau très déformé en voie de subir la fission ternaire.

Les travaux déjà cités de Remy ont montré que cette séparation entre événements $\pi$ et $\pi$ permettait de rapprocher les événements $\pi$ des événements ternaires ou d'ordre supérieur, l'impulsion transverse vue dans le détecteur ne résultant pas d'un transfert d'énergie mais de l'impulsion manquante de la ou des particules émises à l'instant de la désintégration, et non enregistrables du fait de leur perte d'énergie linéique trop faible. D'autre part, les propriétés de ces événements, tant du point de vue de leur section efficace en fonction de l'énergie d'excitation [18] que de leur énergie seraient à rapprocher des fragments déficients en neutrons trouvés par les radiochimistes $[19,20]$ lors de bombardements de cibles lourdes par des protons d'énergie $>0,6 \mathrm{GeV}$. Sur la figure 4 , on présente les distributions des rapports de masse pour les deux types d'événements. On note que le rapport du nombre d'événements $\pi$ sur le nombre total d'événements binaires diminue en fonction de la masse de la cible. Il est de 0,5 pour ${ }^{238} \mathrm{U}$ et 0,7 pour ${ }^{197} \mathrm{Au}$.

Sur la figure 4 , nous voyons que les déviations standard des distributions symétrisées des rapports $\mathrm{MH} / \mathrm{ML}$ décroissent lorsque la masse de la cible augmente, tendance valable pour les deux types d'événements $\pi$ et $\not$. Pour chaque cible $\sigma(\mathrm{MH} / \mathrm{ML})$ des événements $\not$ est plus grande que celle d'événements $\pi$. De ces observations, nous remarquons que les noyaux lourds comme $U$ et $T h$ fissionnent statistiquement avec un même pourcentage d'événements $\pi$ et $\hbar$. La distribution des rapports des masses d'événements $\pi$ donne une allure de fission symétrique. alors que les événements $\hbar$ montrent une contribution de fission asymétrique qui est un peu plus grande pour le thorium que pour l'uranium.

Dans la région des noyaux relativement légers $(\mathrm{Bi}, \mathrm{Pb}, \mathrm{Au})$ la distribution $(\mathrm{MH} / \mathrm{ML})$ des événements $\pi$ s'élargit, et l'élargissement augmente vers le noyau plus léger $(\mathrm{Au})$, ce qui signifie que pour ces noyaux, les événements $\pi$ ne présentent pas l'aspect tout à fait symétrique de division en masse et que la contribution asymétrique n'est plus négligeable. Pour les événements $\not h$, la fission asymétrique est la plus importante.

Des résultats à peu près semblables ont été obtenus par la méthode radiochimique, les produits d'interaction du $\mathrm{Pb}$ avec les protons de $3 \mathrm{GeV}$ se distribuent dans un domaine assez large de masse, et les rendements de ces produits ne varient pas beaucoup dans les régions de la fission et de la spallation [21]. Une partie des événements binaires du type $\alpha_{12} \neq \pi$ provient d'une rupture du noyau en plus de deux fragments et serait donc des événements ternaires dont un des fragments ne serait pas enregistré. Etudions maintenant les propriétés de ces événements ternaires.

4.4.2 Evénements ternaires. - Comme dans le cas des événements binaires, nous faisons une étude géométrique et cinématique des événements ternaires. Nous ne considérerons que les événements dont les trois branches présentent des longueurs supérieures à $5 \mu$. Les principaux résultats sont reportés sur le tableau III.

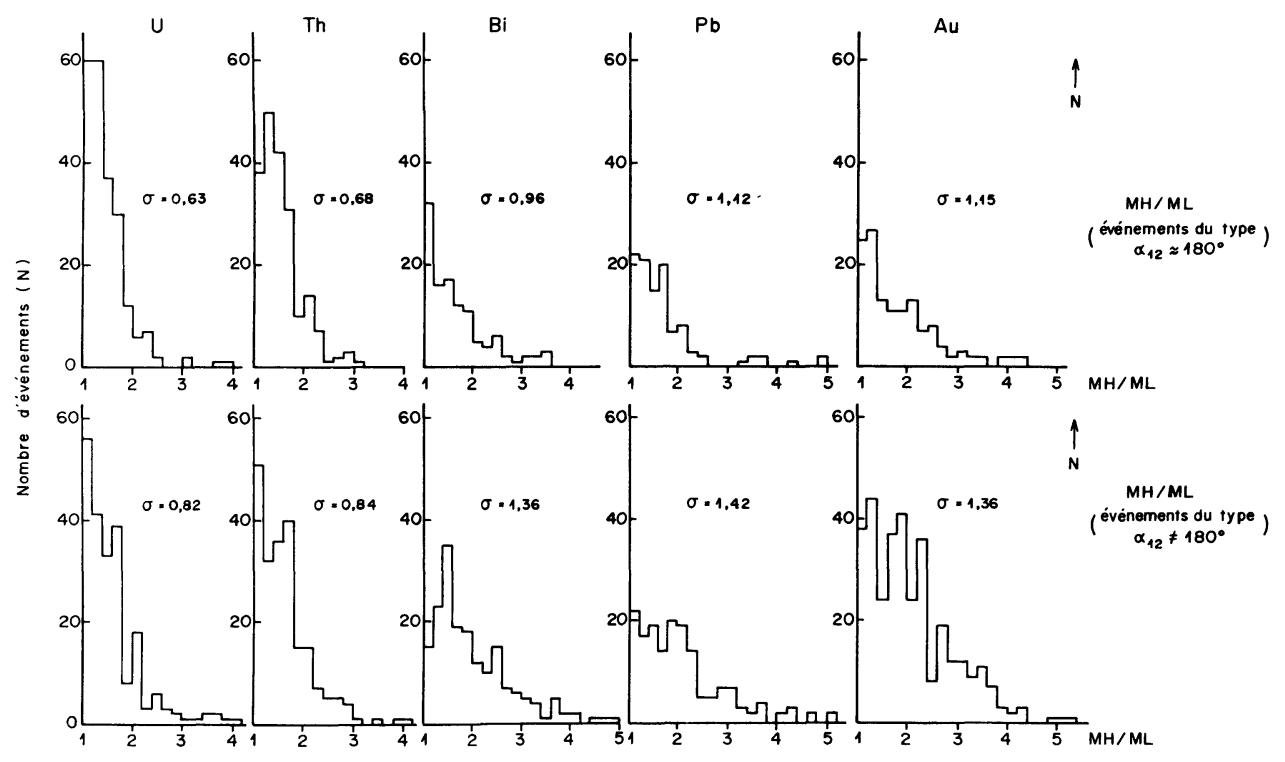

FIG. 4. - Distribution des rapports de masse pour les événements $\pi$ et $九$. 
TABLEAU III

Résultats pour les fissions ternaires

[Results concerning ternary fission]

\begin{tabular}{|c|c|c|c|c|c|c|c|c|c|c|c|c|c|c|}
\hline Cible & Fragment & $\begin{array}{l}\text { the d'éven }^{\text {ts }} \\
\text { calculés }\end{array}$ & $\bar{R}(\mu)$ & $\sigma_{R}$ & $\bar{\alpha}$ (degré) & $\sigma_{\alpha}$ & $\bar{\theta}_{\mathrm{CM}}$ (degré) & $\sigma_{\theta \mathrm{C} . \mathrm{M}}$ & $\bar{M}$ & $\sigma_{M}$ & $\bar{E}_{\mathrm{C} . \mathrm{M}}$ & $\sigma_{\mathrm{E}}$ & $\bar{Q}$ & $\sigma_{Q}$ \\
\hline \multirow{2}{*}{-} & - & - & - & - & - & - & - & - & - & - & - & - & - & - \\
\hline & $\mathrm{L}$ & & 15,6 & 6,4 & $\alpha_{L M}=103$ & 31 & $\theta_{\mathrm{LM}}=109$ & 25 & 37 & 9 & 34 & 20 & & \\
\hline \multirow[t]{3}{*}{$\mathrm{Au}^{197}$} & $\mathbf{M}$ & 132 & 16,9 & 6,8 & $\alpha_{\mathrm{MH}}=146$ & 30 & $\theta_{\mathrm{MH}}=140$ & 20 & 58 & 13 & 51 & 30 & 107 & 15 \\
\hline & $\mathrm{H}$ & & 11,3 & 5,2 & $\alpha_{\mathrm{HL}}=110$ & 37 & $\theta_{\mathrm{HL}}=110$ & 24 & 104 & 17 & 33 & 26 & & \\
\hline & $\mathrm{L}$ & & 16,1 & 6,3 & $\alpha_{\mathrm{LM}}=104$ & 37 & $\theta_{\mathbf{L M}}=105$ & 29 & 32 & 11 & 29 & 22 & & \\
\hline \multirow[t]{3}{*}{$\mathrm{Pb}$} & $\mathbf{M}$ & 71 & 16,2 & 6,1 & $\alpha_{\mathrm{MH}}=155$ & 35 & $\theta_{\mathrm{MH}}=145$ & 19 & 58 & 17 & 43 & 18 & 109 & 24 \\
\hline & $\mathrm{H}$ & & 9,4 & 3,9 & $\alpha_{\mathrm{LH}}=102$ & 37 & $\theta_{\mathbf{L H}}=109$ & 29 & 120 & 22 & 24 & 17 & & \\
\hline & $\mathrm{L}$ & & 18,4 & 7,1 & $\alpha_{\mathrm{LM}}=104$ & 47 & $\theta_{\mathbf{L M}}=100$ & 28 & 35 & 13 & 40 & 29 & & \\
\hline \multirow[t]{3}{*}{$\mathrm{Bi}$} & M & 44 & 19,2 & 7,5 & $\alpha_{\mathrm{MH}}=147$ & 36 & $\theta_{\mathrm{MH}}=147$ & 17 & 59 & 15 & 56 & 25 & 118 & 24 \\
\hline & $\mathrm{H}$ & & 12,5 & 5,0 & $\alpha_{\mathrm{HL}}=96$ & 47 & $\theta_{\mathrm{HL}}=113$ & 28 & 117 & 23 & 39 & 26 & & \\
\hline & $\mathrm{L}$ & & 16,6 & 5,7 & $\alpha_{\mathrm{LM}}=112$ & 29 & $\theta_{\mathrm{LM}}=106$ & 20 & 44 & 12 & 41 & 21 & & \\
\hline \multirow[t]{3}{*}{$\mathrm{Th}^{232}$} & M & 38 & 18,9 & 6,3 & $\alpha_{\mathrm{MH}}=150$ & 34 & $\theta_{\mathrm{MH}}=143$ & 17 & 73 & 14 & 67 & 34 & 174 & 22 \\
\hline & $\mathrm{H}$ & & 13,8 & 6,1 & $\alpha_{\mathrm{HL}}=98$ & 43 & $\theta_{\mathrm{HL}}=110$ & 21 & 117 & 20 & 48 & 32 & & \\
\hline & $\mathrm{L}$ & & 17,8 & 8,8 & $\alpha_{\mathrm{LM}}=106$ & 45 & $\theta_{\mathrm{LM}}=110$ & 32 & 38 & 14 & 39 & 24 & & \\
\hline \multirow[t]{2}{*}{$\mathrm{U}$} & M & 65 & 17,1 & 6,5 & $\alpha_{\mathrm{MH}}=145$ & 24 & $\theta_{\mathrm{MH}}=143$ & 24 & 71 & 19 & 57 & 31 & 175 & 31 \\
\hline & $\mathrm{H}$ & & 11,5 & 4,8 & $\alpha_{\mathrm{HL}}=98$ & 47 & $\theta_{\mathrm{HL}}=106$ & 32 & 132 & 25 & 35 & 30 & & \\
\hline
\end{tabular}

Le calcul des événements ternaires est effectué sur le même principe que celui des événements binaires, à partir des parcours des fragments et des cosinus directeurs des traces correspondantes.

Les fragments sont classés en léger (L), moyen (M) et lourd $(\mathrm{H})$. Le tableau III résume les résultats obtenus et montre les valeurs moyennes et les déviations standard des distributions des parcours $\left(\bar{R}_{i}, \sigma_{R_{i}}\right)$, des angles projetés $\left(\bar{\alpha}_{i j}, \sigma_{\alpha i j}\right)$, des angles $\left(\theta_{i j}, \sigma_{\theta_{i j}}\right.$ ) entre les directions d'émission des fragments $(i)$ et $(j)$ dans le système du centre de masse du noyau fissionnant (ici, noyau composé), des masses $\left(\bar{M}_{i}, \sigma_{M_{i}}\right)$, des énergies dans le système du centre de masse $\left(E_{i}, \sigma_{E_{1}}\right)$ et des énergies de masse libérées lors de la division du noyau composé en trois fragments $\left(\bar{Q}, \sigma_{Q}\right)$, $[i \neq j, i, j=\mathrm{L}, \mathrm{M}, \mathrm{H}]$.

On voit que, pour chaque cible, le parcours du fragment moyen est le plus long, et celui du fragment le plus lourd est le plus court. Les parcours moyens des fragments augmentent avec la masse du noyau cible.

Les déviations standard des distributions en parcours sont deux fois plus grandes que dans le cas des événements binaires, mais les trois distributions relatives aux fragments lourds, moyens et légers présentent des déviations standard très proches. Les distributions angulaires $\left(\alpha_{i j}\right)$ sont également plus larges que celles des événements binaires (de même celles des événements binaires $\not$ ).

On remarque dans les différentes distributions des angles projetés $\alpha_{i j}$ que l'angle $\alpha_{\mathrm{MH}}$ est le plus grand $\left(\bar{\alpha}_{\mathrm{MH}} \sim 147^{\circ}\right)$. Ceci est aussi le cas de l'angle $\theta_{\mathrm{MH}}$ entre les trajectoires des fragments moyen et lourd dans le système du centre de masse du noyau fissionnant (ici, noyau composé).

Les angles $\bar{\alpha}_{\mathrm{MH}}$, et $\bar{\theta}_{\mathrm{MH}}$ expriment que les fragments moyen et lourd sont émis dans le système du centre de masse dans le sens presque opposé $\left(\theta_{\mathrm{MH}} \approx 150^{\circ}\right)$. Les fragments légers, dont les angles d'émission par rapport aux lignes de vol des fragments lourd et moyen dans le système du centre de masse sont proches de $100^{\circ}$, pourraient être émis dans la région comprise entre les deux fragments les plus lourds (au col) juste au moment ou immédiatement après la scission. Donc, il semble exister une analogie avec l'émission des particules alpha au cours de la tripartition des noyaux lourds d'uranium induite par des neutrons thermiques, par exemple. Les valeurs $\bar{\theta}_{\mathrm{LM}}, \bar{\theta}_{\mathrm{LH}}$ indiquent que le fragment léger est émis presque perpendiculairement à la ligne de vol des fragments moyen et lourd dans le système de leur centre de masse. Pour les particules alpha, l'angle d'émission le plus probable avec le fragment de fission léger (dans notre cas, fragment moyen) dans le système du laboratoire est égal à $83^{\circ}$ [22].

Les courbes de distribution en masse des fragments légers (L), moyens (M) et lourds $(\mathrm{H})$ sont indiquées sur la figure 5. Les valeurs présentées sont déduites du calcul admettant l'hypothèse du noyau composé. Elles ne servent que comme valeurs de référence, et sont liées étroitement à la masse du noyau de recul introduite dans le calcul. Cependant l'introduction d'une masse initiale inférieure due à l'existence de la cascade intranucléaire n'affectera que très peu la masse moyenne des fragments légers mais surestimera par contre les masses de fragments moyens et lourds. Les distributions en masse des fragments légers présentent un pic caractérisé par une coupure vers les masses faibles au voisinage de $25 \mathrm{UMA}$, ce qui montre que la fréquence du phénomène de désintégration ternaire est d'autant plus élevée que la masse du fragment le plus léger est plus faible. Or cette coupure vers les masses faibles est purement technique et liée au seuil d'enregistrement du détecteur. Il semble donc 

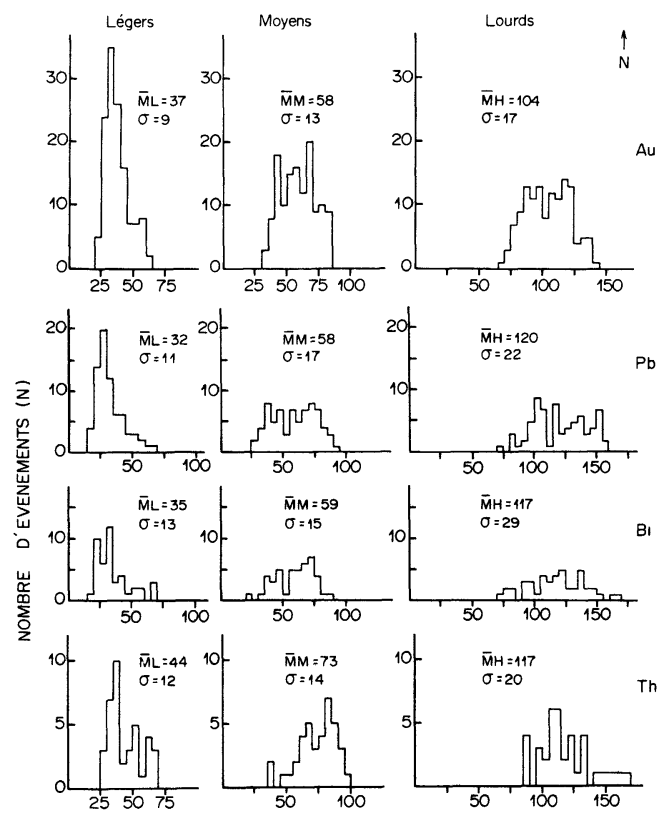

$\mathrm{Pb}$

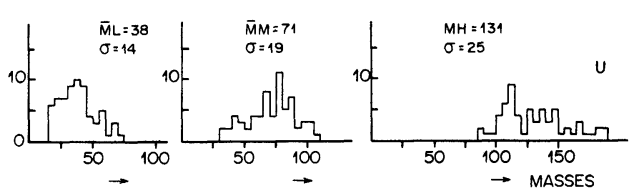

Fig. 5. - Distribution en masses des fragments légers L, moyens M et lourds $\mathrm{H}$.

[Mass yield distribution of light $\mathbf{L}$, middle $\mathbf{M}$, and heavy $\mathrm{H}$ fragments.]

logique de supposer, pour des raisons de continuité, que la production d'événements ternaires mettant en jeu des masses inférieures à 25 UMA et échappant à la détection, existe, et que la fréquence de ce phénomène continue de croître lorsque la masse du fragment le plus léger diminue. De telles désintégrations sont enregistrées sous la forme d'événements binaires de type $\alpha_{12} \neq \pi$.

Les résultats du calcul cinématique des événements ternaires rejoignent les résultats trouvés pour les événements binaires du type $\pi$. L'énergie cinétique des fragments de fission ternaire est reportée sur le tableau III. Les énergies ont été calculées dans le système du centre de masse du noyau fissionnant et sont peu liées aux hypothèses de calcul, puisque déduites, des parcours des fragments. Les valeurs moyennes de l'énergie cinétique totale des fragments (système du centre de masse) sont du même ordre de grandeur que celles correspondant à la fission binaire mais les déviations standard sont nettement plus élevées.

5. Conclusion. - A partir des caractéristiques géométriques des traces des fragments en corrélation, nous avons réalisé l'étude cinématique des fissions binaire et ternaire en supposant la masse du noyau fissionnant égale à la masse du noyau composé. Cette hypothèse certainement inexacte nous a permis de donner des résultats qui ne dépendent que très peu de cette hypothèse : distribution des rapports de masse des fragments ainsi que celle de l'énergie totale. Une séparation entre les événements binaires ayant un angle projeté $\alpha_{12}$ voisin de $180^{\circ}$ et ceux ayant un angle $\alpha_{12} \neq \pi$, nous a permis de montrer une similitude entre cette dernière classe et les événements ternaires. D'une part, l'énergie cinétique totale moyenne mise en jeu dans les désintégrations ternaires, qui est à peine de 10 à $20 \%$ supérieure à celle trouvée dans les désintégrations binaires, semble exclure une contribution appréciable de l'énergie d'excitation du noyau de recul à l'énergie cinétique des fragments. Donc cette énergie d'excitation primaire servirait à l'évaporation de particules et en particulier de neutrons. D'autre part, l'énergie cinétique moyenne des fragments moyens et lourds pour ces deux groupes d'événements est faible comparée à l'énergie cinétique des fragments de fission binaire de type $\alpha_{12} \simeq \pi$.

Tous ces résultats semblent indiquer :

1) qu'une fraction au moins des événements binaires du type $\alpha_{12} \neq \pi$ et que les événements ternaires procèdent d'un même type de désintégration ;

2) que ces deux types d'événements pourraient expliquer une partie des isotopes légers et lourds déficients en neutrons trouvés par les méthodes radiochimiques et que l'on attribuait à des produits de fragmentation.

Enfin l'analyse cinématique des événements ternaires nous montre une analogie avec le phénomène connu de la tripartition de noyaux lourds spontanée comme pour le californium ou induite par des neutrons thermiques comme pour l' $U^{235}$.

A savoir :

- une augmentation de la probabilité du processus ternaire ou de tripartition lorsque la masse du fragment léger émis diminue ;

- le rôle symétrique que jouent les fragments moyen et lourd par rapport au fragment léger émis et qui se traduit par une direction d'émission du fragment léger voisine de la bissectrice de l'angle entre les trajectoires des fragments lourd et moyen et légèrement infléchie dans la direction de ce dernier. Ce résultat n'est qu'une autre forme de présentation des propriétés énergétiques qui implique que les impulsions sont équivalentes dans le système du centre de masse.

Cependant, si ces résultats montrent des similitudes avec les résultats trouvés par d'autres auteurs, ils ne peuvent encore expliquer tous les résultats trouvés sur les interactions des particules de haute énergie avec les noyaux lourds. 


\section{Bibliographie}

[1] Rahimi, F., Gheysari, D., Remy, G., Tripier, J., Ralarosy, J., Stein, R. and Debeauvais, M., Phys. Rev. C 8 (1973) 1500 .

[2] Tripier, J., Remy, G., Debeauvais, M., Ralarosy, J. and SteIn, R., Proceedings of the 8th Int. Conf. on nuclear photography and Solid State Detectors (1972) p. 290.

[3] Brandt, R., Carbonara, F., Cieslak, E., Dakowski, M.. Geller, Ch., Piekarz, H., Piekarz, J., Riezler, W. RINZIVILlo, R., SASSI, E., SowINSKI, M. and ZAKRZEWsKI, G., Nucl. Phys. A 90 (1967) 177.

[4] Remsberg, L. P., Plasil, F., Cumming, J. B. and Perlman, M. L., Phys. Rev. C 1 (1970) 265.

[5] RemY, G., Thèse d'Etat, U.L.P. Strasbourg (1974).

[6] Metropolis, M., Rivinstorm, R., Turkewich, A., Miller, J. and Friedlander, J., Phys. Rev. 94 (1954) 727.

[7] Remsberg, L. P., Plasil, F., Cumming, J. B. and Perlman, M. L., Phys. Rev. 187 (1969) 4.

[8] Tripier, J., Remy, G., Ralarosy, J., Debeauvais, M., Stein, R. and Huss, D., Nucl. Instrum. Methods 115 (1974) 29.

[9] KowalsKi, L., Thèse Orsay (1963).
[10] Remsberg, L. P., Plasil, F., Cumming, J. B. and PerlMAN, M. L., Phys. Rev. C 1 (1970) 265.

[11] Peter, J., Thèse d'Etat Paris (1968).

[12] Viola, V. E. and Sikkeland, T., Phys. Rev. 130 (1963) 2044.

[13] Nix, J. R., Nucl. Phys. A 130 (1959) 241.

[14] Hahn, R. L. and Bertini, H. W., Phys. Rev. C 6 (1972) 660.

[15] Friedlander, G., Physics and Chemistry of Fission I.A.E.A. Salzburg, Vol. II (1965) p. 265.

'[16] Postkanzer, A. M., Butler, G. W. and Hyde, E. K., Phys. Rev. C 3 (1971) 882.

[17] Herz, A. J., O'Ceallaigh, C., Sullivan, D. O. and Thomson, A., Nuovo Cimento A 33 (1976) 487.

[18] Friedlander, G., Friedman, L., Gordon, B. and Yaffe, L.. Phys. Rev. 129 (1963) 1809.

[19] Beg, K. and Porile, N. T., Phys. Rev. C 3 (1971) 1631.

[20] Lagarde, M., Thèse d'Etat, Bordeaux (1972).

[21] Miller, J. and Hudis, J., Annu. Rev. Nucl. Sci. 9 (1959) 159.

[22] Carles, C., Asghar, M., Doan, T. P. and Chastel, R.. Second I.A.E.A. Symposium on Physics and Chemistry of fission, Vienna (1969) 119. 